\title{
Responses of Tonically Active Neurons in the Monkey Striatum Discriminate between Motivationally Opposing Stimuli
}

\author{
Sabrina Ravel, Eric Legallet, and Paul Apicella \\ Institut de Neurosciences Physiologiques et Cognitives, Centre National de la Recherche Scientifique, 13402 Marseille cedex 20, France
}

\begin{abstract}
The striatum is involved in the control of appetitively motivated behavior. We found previously that tonically active neurons (TANs) in the monkey striatum show discriminative responses to different stimuli that are appetitive or aversive. However, these differential responses may reflect the sensory qualities of the stimulus rather than its motivational value. In the present study, we sought to define more precisely the relationship between the particular aspect of the response of TANs and the motivational value of stimuli. For this purpose, three monkeys were presented with two types of aversive stimuli (loud sound and air puff) and one appetitive stimulus (fruit juice). In most instances, the TAN responses to the loud sound and the air puff were similar, in terms of response pattern and duration, whereas responses to the liquid reward showed distinct features. Using classical appetitive conditioning, we reversed the motivational value of a stimulus so that a previously aversive stimulus was now associatively paired with a reward and found that this manipulation selectively modifies the expression of TAN responses to the stimulus. These data indicate that the characteristics of neuronal responses undergo modifications when the valence of the stimulus is changed from aversive to appetitive during associative learning, suggesting that TANs may contribute to a form of stimulus encoding that is dependent on motivational attributes. The adaptation of TAN responses such as observed in the present study likewise reflects a neuronal system that adjusts to the motivational information about environmental events.
\end{abstract}

Key words: striatum; basal ganglia; TANs; motivation; learning; monkey

\section{Introduction}

The striatum, a major component of the basal ganglia, is thought to be engaged in the integration of reward-related information in the brain, receiving input from cortical and limbic regions that may be further modulated by dopaminergic projections from the midbrain. Two main classes of striatal neurons have been identified using single-neuron recording experiments in behaving monkeys. They are the phasically and tonically active neurons (TANs). Phasically active neurons exhibit increases in firing in relation to the detection and expectation of reward (Rolls et al., 1983; Hikosaka et al., 1989; Apicella et al., 1991, 1992; Schultz et al., 1992; Williams et al., 1993; Bowman et al., 1996; Shidara et al., 1998). There is also evidence that these neurons may display different levels of activity depending on which type of reward is obtained at the end of a trial (Hassani et al., 2001). Furthermore, reward information may influence the various forms of neuronal activity that are related to the planning and initiation of behav-

\footnotetext{
Received May 9, 2003; revised July 15, 2003; accepted July 18, 2003.

This work was supported by Centre National de la Recherche Scientifique and Biomed II Program of the European Commission Grant BMH4-CT95-0608. We thank W. Schultz for comments and suggestions on a previous version of this manuscript. We also thank R. Massarino and C. Wirig for expert technical assistance.

Correspondence should be addressed to Paul Apicella, Institut de Neurosciences Physiologiques et Cognitives, Centre National de la Recherche Scientifique, 31 chemin Joseph Aiguier, 13402 Marseille cedex 20, France. E-mail apicella@Incf.cnrs-mrs.fr.

S. Ravel's present address: Laboratory of Neuropsychology, National Institute of Mental Health, Bethesda, MD 20892.

Copyright $\odot 2003$ Society for Neuroscience $\quad$ 0270-6474/03/238489-09\$15.00/0
}

ioral reactions (Hollerman et al., 1998; Kawagoe et al., 1998; Takikawa et al., 2002). On the basis of these findings, it has been argued that the striatum, in particular its ventral part, is involved in behavior that is motivated by rewarding or appetitive stimuli. On the other hand, very few neurophysiological experiments have examined the effects of aversive aspects of motivation on the activity of phasically active striatal neurons (Rolls et al., 1983; Williams et al., 1993; White et al., 1994; Koyama et al., 2000).

TANs play an important role in detecting motivationally salient events from the environment (Aosaki et al., 1994b; Apicella et al., 1997). In a previous brief communication, we found that TANs were responsive when monkeys received a rewarding fruit juice and an unpleasant air puff, but the air puff characteristically produced a triphasic response pattern that was very rarely observed with the liquid reward (Ravel et al., 1999). This differential responsiveness is of particular interest, because it suggests that the motivational evaluation of the stimulus can shape TAN responses to the stimulus. However, it is unclear whether such capacity to distinguish among motivationally opposing stimuli reflects a difference in the physical identity of the stimulus or a difference in its motivational value. In particular, it is not known whether TANs can show response plasticity when the motivational value of sensory information undergoes changes into opposite directions during associative learning.

In this experiment, we explored further to what extent the particular response profile of TANs reflected the evaluation of 
positive or negative valenced stimuli. We also determined whether the characteristics of TAN responses were changed as monkeys became experienced with the associative relationship between a previously aversive stimulus and a rewarding event during classical conditioning. Our findings showing a difference in the expression of neuronal responses to an identical stimulus presented before and after appetitive learning have revealed for the first time the capacity of TANs to adapt their responsiveness to the specific motivational value of stimuli.

\section{Materials and Methods}

Behavioral procedures. We used three male macaque monkeys (Macaca fascicularis; monkeys $\mathrm{C}$, $\mathrm{J}$, and $\mathrm{T}$ ) that were habituated to being seated in a restraining box that was described in a previous publication (Apicella et al., 1997). Animal housing and experimental procedures were in accordance with the National Institutes of Health Guide for the Care and Use of Laboratory Animals and the French laws on animal experimentation.

Initial anesthesia was administered by an intramuscular injection of ketamine (10 mg/kg; Sanofi, Libourne, France), and a deep anesthesia was induced by intravenous injection of pentobarbital sodium $(35 \mathrm{mg} /$ $\mathrm{kg}$; Sanofi) and maintained as needed. Under appropriate aseptic conditions, animals were implanted with a head-holding device, a stainless steel recording chamber, and two pairs of silver-silver chloride electrodes for recording eye movements (Apicella et al., 1997). Outside of any task, monkeys were presented with irregularly timed stimuli having an appetitive or aversive value: a squirt of fruit juice $(0.3 \mathrm{ml})$ delivered to the monkey's mouth, a stream of compressed air (65 psi, $100 \mathrm{msec})$ directed toward the monkey's face, and a loud sound ( $90 \mathrm{~dB}, 50 \mathrm{msec})$ emitted from a distant source inside the experimental room. The juice was delivered via a solenoid valve from a spout mounted in front of the monkey's mouth. The air puff, controlled by a solenoid valve, was delivered from a pipette tip placed $2 \mathrm{~cm}$ away from the monkey's face, at eye level. The valves were placed outside the experimental room to prevent the monkeys from hearing the solenoid noise. The air puff made a $50 \mathrm{~dB}$ noise that was audible to the animal whenever it was delivered. In some instances, we checked the relationship of neuronal responses to this noise alone by disconnecting the tube between the solenoid valve and the pipette tip. This manipulation eliminated the tactile component of the air puff but maintained the auditory component. The stimuli were tested separately in blocks of 30-40 successive trials, and interstimulus intervals varied randomly between 5.5 and $8.5 \mathrm{sec}$. A close-up video system served to continuously monitor the monkey's face. Mouth movements were recorded using force transducers (strain gauges) mounted on the drinking tube. Although the air puff and the loud sound could be tolerated readily, they were aversive enough to ensure overt behavioral signs of monkey discomfort, notably mouth opening, grimacing, and eyeblink responses, as assessed by video monitoring and electrooculographic recordings. We also found that the occasional presentation of the auditory component of the air puff alone was effective in eliciting behavioral reactions similar to those produced by face somatosensory stimulation, suggesting that these reactions were conditioned responses to the air puff. In contrast, the juice constituted an appetitive stimulus for the animal, which reacted with rhythmic licks to the delivery of liquid.

After exposure to aversive and appetitive stimuli, monkeys $\mathrm{C}$ and $\mathrm{J}$ were trained on a classical conditioning procedure that uses an aversive auditory stimulus that was followed, after a fixed interval of $1 \mathrm{sec}$, by the delivery of fruit juice. Both monkeys received daily training sessions consisting of three to six blocks of 30-40 stimulus-reward pairing trials each. This conditioning situation was applied only while a neuron was being recorded, and animals gained experience with the stimulus-reward association as the recording sessions progressed. During acquisition of the stimulus-reward association, blocks of reward-alone trials were interleaved with blocks of conditioning trials to assess the responsiveness to reward given free outside of the learning context.

Neuronal recordings. We used standard electrophysiological methods for recording the extracellular activity of TANs in both the caudate nucleus and putamen. Single-neuron recording was performed with glasscoated tungsten electrodes (exposed tips of 9-12 $\mu \mathrm{m}$ length and $3 \mu \mathrm{m}$ diameter) that were passed inside a guide cannula (outer diameter, 0.6 $\mathrm{mm}$ ) at the beginning of each session. After penetration of the dura, the electrode was advanced toward the striatum with a hydraulic microdrive (MO-95; Narishige, Tokyo, Japan) until the activity of one neuron was isolated. Signals from neuronal activity were amplified, filtered (bandpass, $0.3-1.5 \mathrm{kHz}$ ), and converted to digital pulses through a window discriminator. The recording experiment was controlled online by a computer, which presented stimuli and collected the signals from neuronal activity and analog signals from mouth and eye movements. As described in detail previously (Aosaki et al., 1994b; Apicella et al., 1997), TANs were easily distinguished from other striatal neurons by their specific electrophysiological characteristics. Histological locations of recording sites were verified at the termination of data collection in all monkeys.

Data analysis. The behavioral assessment of stimulus-reward associative learning was the initiation of licking movements produced by the presentation of the auditory stimulus. We calculated the frequency of trials during which licking movements started before the delivery of reward, and we measured the latency of these movements relative to the onset of the auditory stimulus. The entire period of training for each monkey was divided into four phases. The early phase corresponded to a period in which all recorded TANs responding to the auditory stimulus showed a type of response specific of an aversive stimulus. It was only after 14 blocks (day 4) and 29 blocks (day 8) of stimulus-reward pairing trials that a TAN first showed a type of response to the auditory stimulus that is specific of an appetitive stimulus in monkeys $\mathrm{C}$ and J, respectively. The duration of each of the subsequent phases was chosen in an attempt to equate the number of tested neurons in each monkey, with the exception of monkey J, in which the last period was continued for $24 \mathrm{~d}$ (60 blocks) to test for the long-term stability of the neuronal responses to the conditioned stimulus. Data are therefore separated according to the training period [first period: monkey C, $4 \mathrm{~d}$ (14 neurons); monkey J, $8 \mathrm{~d}$ (29 neurons); second period: monkey C, $4 \mathrm{~d}$ (16 neurons); monkey J, $6 \mathrm{~d}$ (22 neurons); third period: monkey C, $7 \mathrm{~d}$ (23 neurons); monkey J, $5 \mathrm{~d}$ ( 24 neurons); fourth period: monkey C, $11 \mathrm{~d}$ (23 neurons); monkey J, $24 \mathrm{~d}$ (60 neurons)].

Neuronal responsiveness was evaluated in terms of proportions of responding neurons. A standard time-window procedure based on a Wilcoxon signed rank test served to assess the statistical significance $(p<0.05)$ of changes in neuronal firing rate after the onset of a stimulus for each neuron (Apicella et al., 1997). The latency and duration of all neuronal responses were determined with this procedure and from inflections in cumulative frequency distributions. Differences in proportions of responding neurons among testing conditions were assessed by using the $\chi^{2}$ test, and neuronal response durations were compared between different conditions or different phases of training with one-way ANOVA. For the response duration analysis, recorded neurons were discarded when data were contaminated by noises or artifacts. In addition to the assessment of responses of the individual neurons, the overall responsiveness of TANs was visually compared between different phases of training using population response histograms constructed by averaging activity of all TANs recorded in each period, independent of the presence or absence of a response (Aosaki et al., 1994b; Ravel et al., 2001). For each neuron, a normalized perievent time histogram was obtained by dividing the content of each bin by the number of trials, and the population histogram was obtained by averaging all normalized histograms.

\section{Results}

In the initial phases of our experimentation, the activity of 127 TANs in the striatum of the three monkeys was recorded during exposure to motivationally opposing stimuli. We found that the great majority of these neurons responded to the loud sound (22 of 24 neurons, $92 \%$ in monkey C; 46 of 50, 92\% in monkey J; 47 of $53,89 \%$ in monkey $\mathrm{T}$ ). These responses did not decrease over time with repeated exposures to the stimulus, suggesting a lack of habituation. Most TANs also responded to the rewarding liquid ( 21 of 24 neurons, $88 \%$ in monkey C; 45 of 50, 90\% in monkey J; 48 of $50,96 \%$ in monkey T) and to the aversive air puff ( 20 of 23 neurons, $87 \%$ in monkey C; 21 of $27,78 \%$ in monkey J; 43 of 48, 

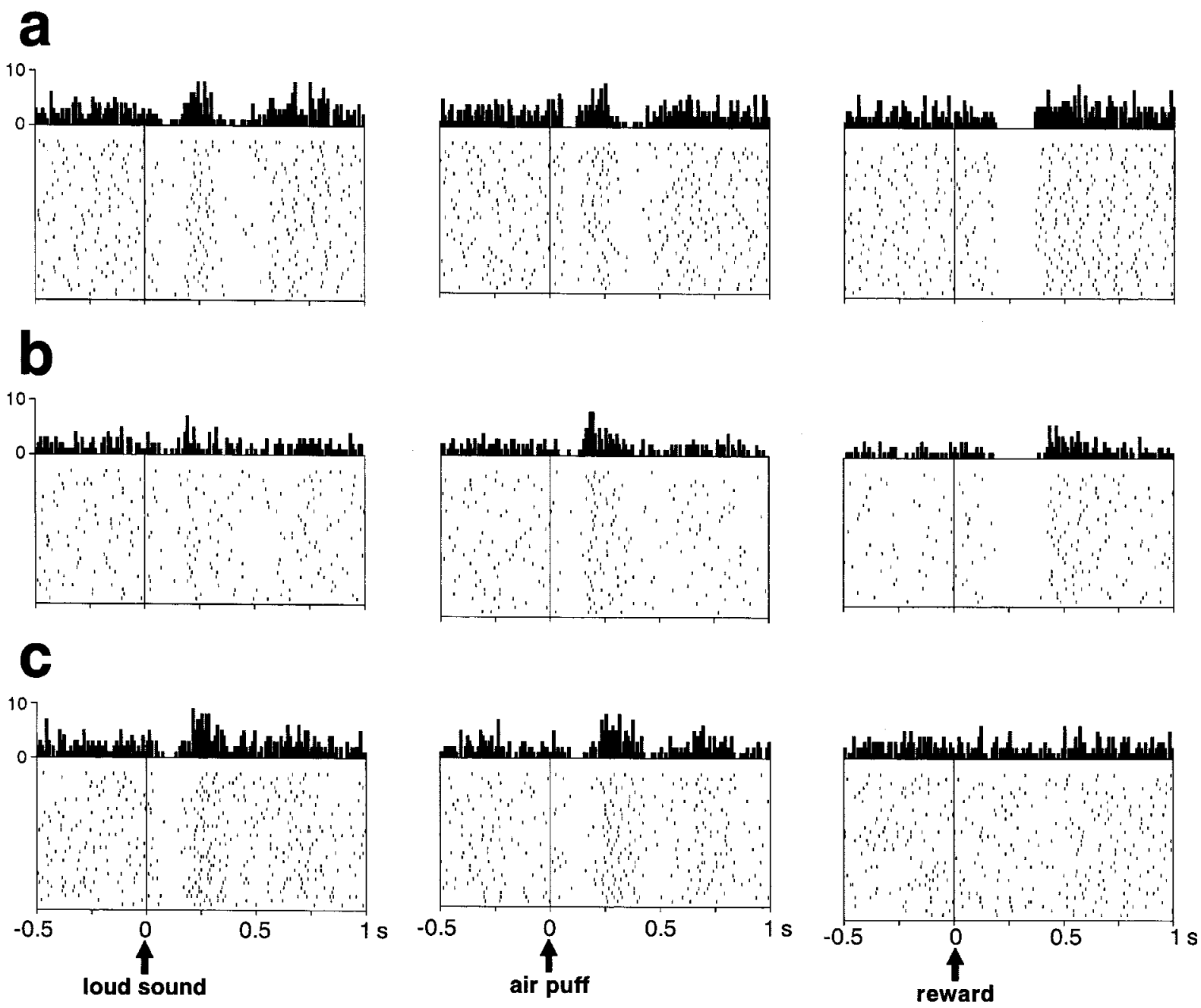

Figure 1. Responses to aversive and appetitive stimuli in three TANs. Each dot represents a neuronal impulse, and each line of dots represents the neuronal activity recorded during a trial. Histograms and dot displays of neuronal activity are aligned on the onset of stimuli. The neurons for which the activity is shown in $a$ and $b$ responded to all three stimuli but displayed a different pattern of responses between aversive (left and middle) and appetitive (right) stimuli. The response to the air puff of the neuron shown in $b$ was larger than to the loud sound. One example of a neuron responding selectively to aversive stimuli is shown in c. Vertical calibration on histograms is in impulses per bin. Bin width for histograms is $10 \mathrm{msec}$.

90\% in monkey T), consistent with previous findings (Ravel et al., 1999). There were no significant differences in the fractions of responding TANs among the three stimuli used $\left(p>0.05 ; \chi^{2}\right.$ test). A total of 96 neurons in our sample were each tested with the three stimuli, and $74(77 \%)$ maintained responses to all of them (Fig. $1 a, b$ ). In some cases, as illustrated in Figure $1 b$, the responses to aversive stimuli varied in terms of magnitude of the changes in activity, but it is not known whether this response modulation depended on differences in the sensory characteristics of the stimulus being presented or on differences in its aversive impact. There also appears to be at least some degree of response specificity in 22 of the 96 neurons (23\%) tested with all three stimuli. Six neurons responded selectively to aversive stimuli. An example of such a neuron is shown in Figure $1 c$. Only three neurons responded selectively to reward. Thirteen neurons responded to both one aversive stimulus (air puff, four neurons; loud sound, nine neurons) and reward.

It is notable that neuronal responses to the air puff and the loud sound were similar, whereas TANs responded differently to reward. As shown in Figure 1, $a$ and $b$, a number of neurons responding to aversive stimuli exhibited a depression in firing, followed by an activation and then by another phase of depres- sion (loud sound, 49 of 115 neurons, 43\%; air puff, 39 of 84, $46 \%$ ), whereas most responses to reward consisted of a depression that was followed by an activation ( 96 of 114 neurons, $84 \%$ ). Moreover, durations of the initial depression in activity differed significantly between appetitive and aversive stimuli, being longer for reward $(161 \pm 53 \mathrm{msec}$, mean $\pm \mathrm{SD} ; n=114)$ than for the air puff $(78 \pm 39 \mathrm{msec} ; n=78)$ and loud sound $(81 \pm 25$ msec; $n=113$ ). The average histograms for the responses of all TANs recorded in monkeys $\mathrm{C}$ and $\mathrm{J}$, which were subsequently trained on the classical conditioning procedure, are shown in Figure 2. It appears that contrasting response features of individual neurons for aversive and appetitive stimuli were sufficiently strong to result in qualitatively different response profiles of the whole population.

Although these results suggest the existence of differences in the encoding of specific motivational attributes, it is possible that the differential responses of TANs to pleasant and unpleasant stimuli are correlated with the capacity of these stimuli to elicit distinct behavioral reactions. To determine whether particular neuronal responses may have been related selectively to particular reactions from the animal, such as eye movements occurring in the aversive stimulus conditions, we compared the responses 

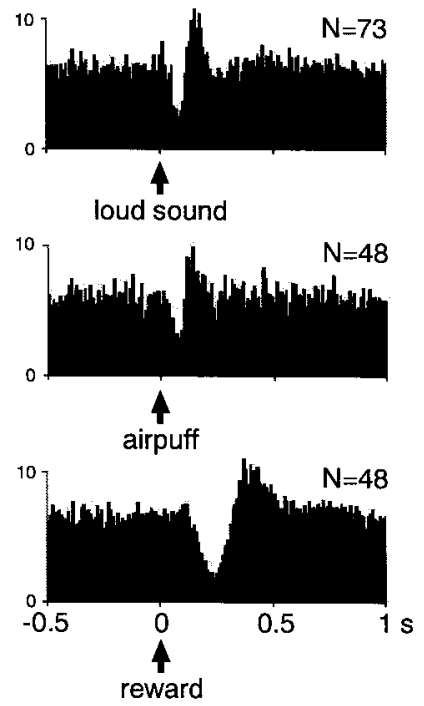

Figure 2. Population response histograms of TANs tested with aversive and appetitive stimuli. $N$, Number of neurons included for each histogram.

of TANs when the loud sound produced eye movements with those evoked in the absence of eye movements. The results of this analysis are shown for a neuron in Figure $3 a$. For most TANs, like this one, the response to the stimulus was not linked with the occurrence of a behavioral reaction at each trial, thus suggesting that responses were not related to the initiation of individual eye movements. Another possibility is that the responses of TANs reflect the probability that a stimulus will elicit a behavioral reaction rather than each initiation of a movement, as suggested by Blazquez et al. (2002). We therefore examined whether the responsiveness of TANs to aversive stimuli was influenced by the frequency of animals' reactions to these stimuli. In monkey J, a sufficient amount of data to analyze was collected for neurons whose activity was recorded with the loud sound and the faint noise without air-puff delivery. The results are shown in Figure $3 b$. For this animal, the frequency of eye movements elicited by the stimuli was similar for the loud sound ( $44 \pm 9 \%)$ and the faint noise $(45 \pm 10 \%)$ (Fig. 3b, left), whereas the fraction of responding neurons was significantly higher $\left(\chi^{2}=4.86\right.$; $\left.\mathrm{df}=1 ; p<0.05\right)$ with the loud sound than with the faint noise (Fig. $3 b$, middle). The population activity for each aversive stimulus shows that the average response to the aversive stimulus was more pronounced for the loud sound than to the faint noise (Fig. $3 b$, right), suggesting that differences in the responsiveness of TANs were not paralleled by differences in the frequency of eye movement reactions.

The positions of the 96 neurons tested with the loud sound, the air puff, and the liquid reward are shown in Figure 4. The majority of these neurons were located throughout the entire putamen, including its posterior part, which is considered as the motor-related region of the striatum (Parent, 1990), with only a small number recorded in the caudate nucleus. There was no apparent spatial clustering of TANs with responses to aversive and/or appetitive stimuli. It is worth noting that the presently observed neuronal responses were not located in the ventral parts of the caudate nucleus and putamen rostral to the anterior commissure, which receive projections from limbic structures.

Given the neuronal discrimination between motivationally opposing stimuli, we then studied to which extent TANs can change their responsiveness when the motivational value of a stimulus is reversed. To this aim, we used, for monkeys $\mathrm{C}$ and J, a classical conditioning procedure in which an aversive stimulus was paired with liquid reward delivery. For monkey J, the stimulus was the loud sound, whereas for monkey C, it was the auditory component of the air puff. The behavioral expression of the acquired stimulus-reward association is illustrated by the learning curves of Figure 5. In the early stage of conditioning, the percentage of licks preceding reward delivery remained relatively constant, albeit some modest decline occurred during the second period. Anticipatory licking started increasing gradually over the third period of training, indicating that the monkey used this stimulus as an effective predictor that reward will be delivered. On the other hand, it was only later that latencies of anticipatory licks began to decrease, indicating that the two behavioral indices we used to assess learning changed with different time courses across the training blocks: the formation of the stimulus-reward association occurred more quickly than the development of response timing. A dissociation between the correct selection of motor responses and the speed of performance has been also reported during the learning of sequential movement procedures (Hikosaka et al., 1995).

In the two monkeys, a total of 211 neurons were recorded during the stimulus-reward pairing (76 from monkey C; 135 from monkey J). The first learning change consisted in a modification in the manner in which neurons responded to the auditory stimulus when the animals' behavior adapted to the new motivational significance of this stimulus. Figure $6 a$ shows typical examples of responsive TANs recorded as learning progressed. There was a decrease in the proportion of neurons producing triphasic responses (first period, 13 of $31,42 \%$; fourth period, 11 of 81 , $\left.14 \% ; \chi^{2}=10.76 ; \mathrm{df}=1 ; p<0.01\right)$, and durations of the initial depression in activity were lengthened (first period, $110 \pm 49$ msec, $n=31$; fourth period, $176 \pm 61 \mathrm{msec}, n=81 ; F_{(1,109)}=$ 27.99; $p<0.01$; one-way ANOVA, followed by Fisher's test). The second learning change consisted in a changed selectivity of the neurons responding to the auditory stimulus or to reward. As shown in Figure $6 b$, there was a decline in the proportion of reward selective responses (monkey $\mathrm{C}, \chi^{2}=27.32$, $\mathrm{df}=3, p<$ 0.01 ; monkey $\mathrm{J}, \chi^{2}=12.52, \mathrm{df}=3, p<0.01$ ) paralleled by an increase in the proportion of signal-selective responses across training periods (monkey C, $\chi^{2}=12.78$, $\mathrm{df}=3, p<0.01$; monkey $\mathrm{J}, \chi^{2}=10.79$, df $=3, p<0.05$ ). The third learning change consisted in a sharp reduction in the response to reward during associative learning. Of 43 neurons recorded during the earliest phase of learning in the two monkeys, 35 neurons (81\%) responded to reward, whereas 32 of 83 neurons (39\%) responded to reward on the late phase of learning $\left(\chi^{2}=20.87 ; \mathrm{df}=1\right.$; $p<0.01)$.

To give an overview of the evolution of the responsiveness of the whole population of TANs as the animal learned the stimulus-reward relationship, population responses of all neurons recorded in monkey J are illustrated in Figure 7. Although overall responsiveness to the auditory stimulus was maintained over the entire period of training, a qualitatively different population response emerged when the original stimulus was followed by reward delivery. Instead of the triphasic response pattern occurring during the earliest phase of learning, there was a biphasic response pattern similar to that elicited by reward, suggesting that the appetitive value of the reward has been associated through classical conditioning with the auditory stimulus. There was also an initial predominance followed by a gradual decline in the average response to reward leading, in the late stage of training, to an almost complete disappearance of the response. On the other hand, a robust response to reward given at unpredictable times 

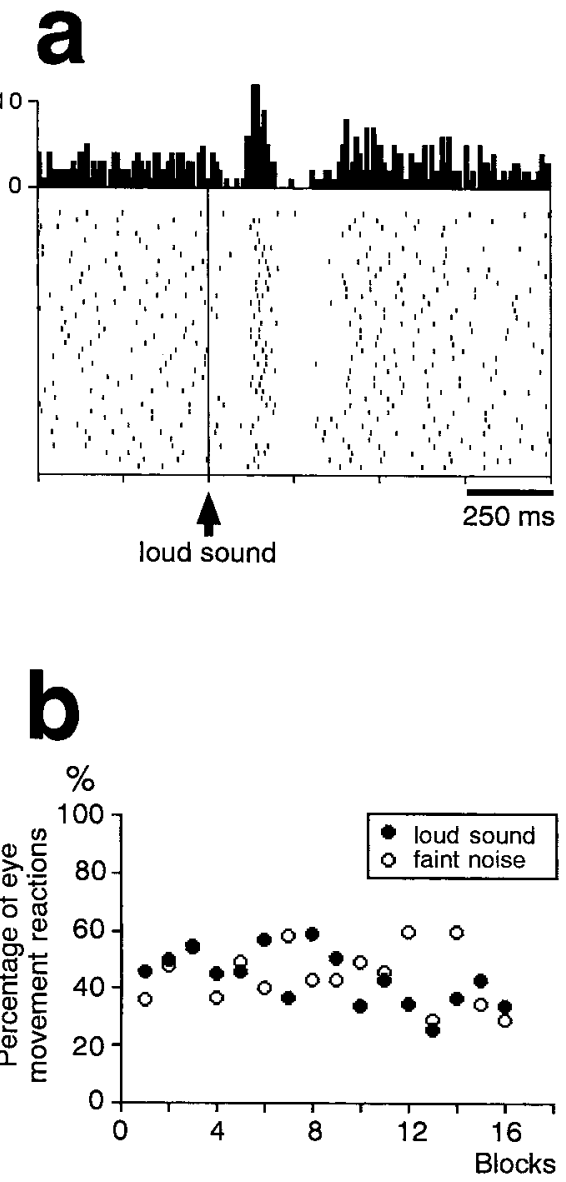

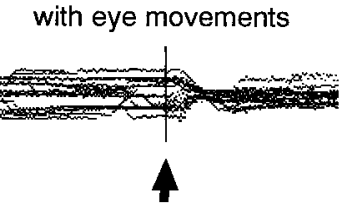

without eye movements

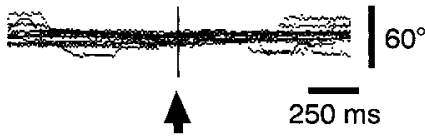

loud sound

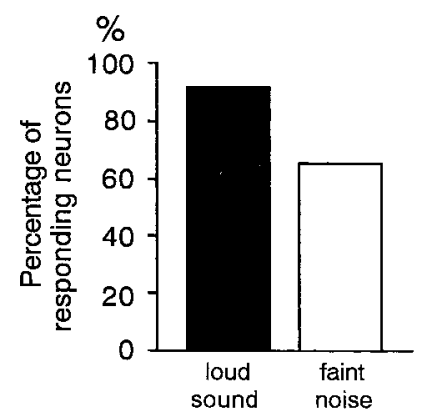

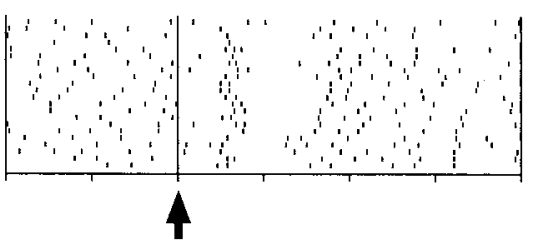

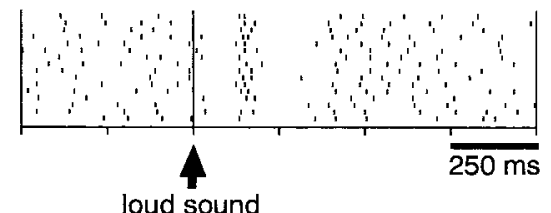

loud sound
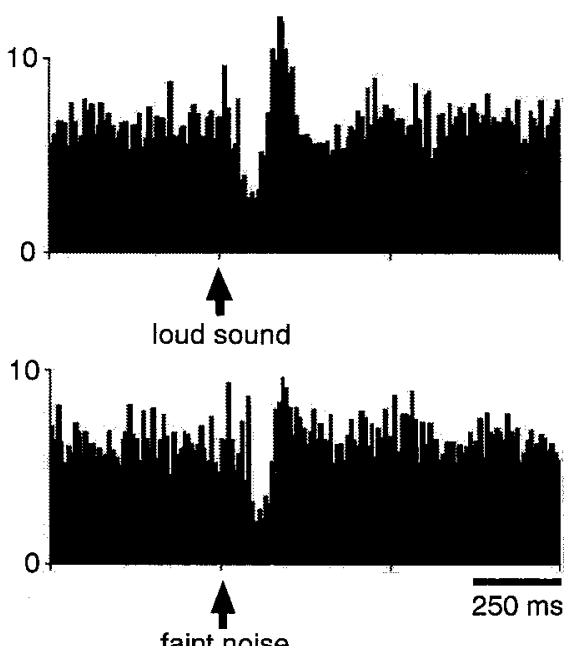

Figure 3. Lack of relation of TAN responses to behavioral reactions evoked by aversive stimuli. $a$, Independence of neuronal responses from individual eye movements elicited by the loud sound in one TAN. Same conventions as in Figure 1. Data were collected when the loud sound was repeatedly presented during a block of trials (left) and were separated offline according to presence or absence of eye movements (right). Superimposed traces of horizontal electrooculograms recorded simultaneously in the same trials (middle). $b$, Percentage of eye movements evoked by two aversive stimuli (left). Separate symbols are used to show block percentage of eye movement reactions recorded for the loud sound and the faint noise associated with air-puff delivery. Total percentage of responding neurons (middle). Population responses of TANs to the two aversive stimuli (right). Number of neurons included for each histogram (middle and right) are as follows: loud sound, 30 neurons; faint noise, 23 neurons.

outside of any task persisted during the entire course of learning in the same neurons (Fig. 7, right).

\section{Discussion}

The present findings show that TANs in the monkey striatum responded in the same manner to the presentation of two types of aversive stimuli, the characteristics of these responses contrasting with those elicited by an appetitive stimulus. To confirm that it is the motivational value of the stimulus and not the type of stimulus per se that is critical for eliciting a particular response profile, we reversed the aversive evaluation of a stimulus by using stimulus-reward association learning. Our data show that the precise form of the response of TANs to an original aversive event is modified when this event acquired conditioned appetitive properties. Thus, the manner in which TANs respond to an identical stimulus presented before and after associative learning seems to reflect the way in which monkeys reevaluate the motivational significance of the stimulus. We believe that this differential expression of TAN responses is compatible with the idea that motivational attributes shape the neuronal responsiveness to sensory events, although alternative interpretations might be found in terms of behavioral reactions and attentional processes associated with stimulus detection.

\section{Nature of TAN responses}

Most studies indicated that TAN responses reflect rewarding components of stimuli rather than motor components (Aosaki et al. 1994b; Apicella et al., 1997). Although the responses of individual neurons we described were unrelated to eye movements elicited by aversive stimuli, another factor possibly contributing to these responses relates to the idea that the overall responsiveness averaged across a population of TANs can be associated with the probability of specific behavioral reactions (Blazquez et al., 2002). However, the present data suggest that, under our experimental conditions, the population response of TANs to an aversive stimulus did not appear to correlate with the frequency of eye movements elicited by this stimulus, suggesting that TAN responses reflect the motivational evaluation of the stimulus, independently of the behavioral reaction itself. It is also possible that the differential expression of TAN responses to appetitive and aversive stimuli may have come from different levels of attention to such stimuli. Previous studies provided evidence that the re- 

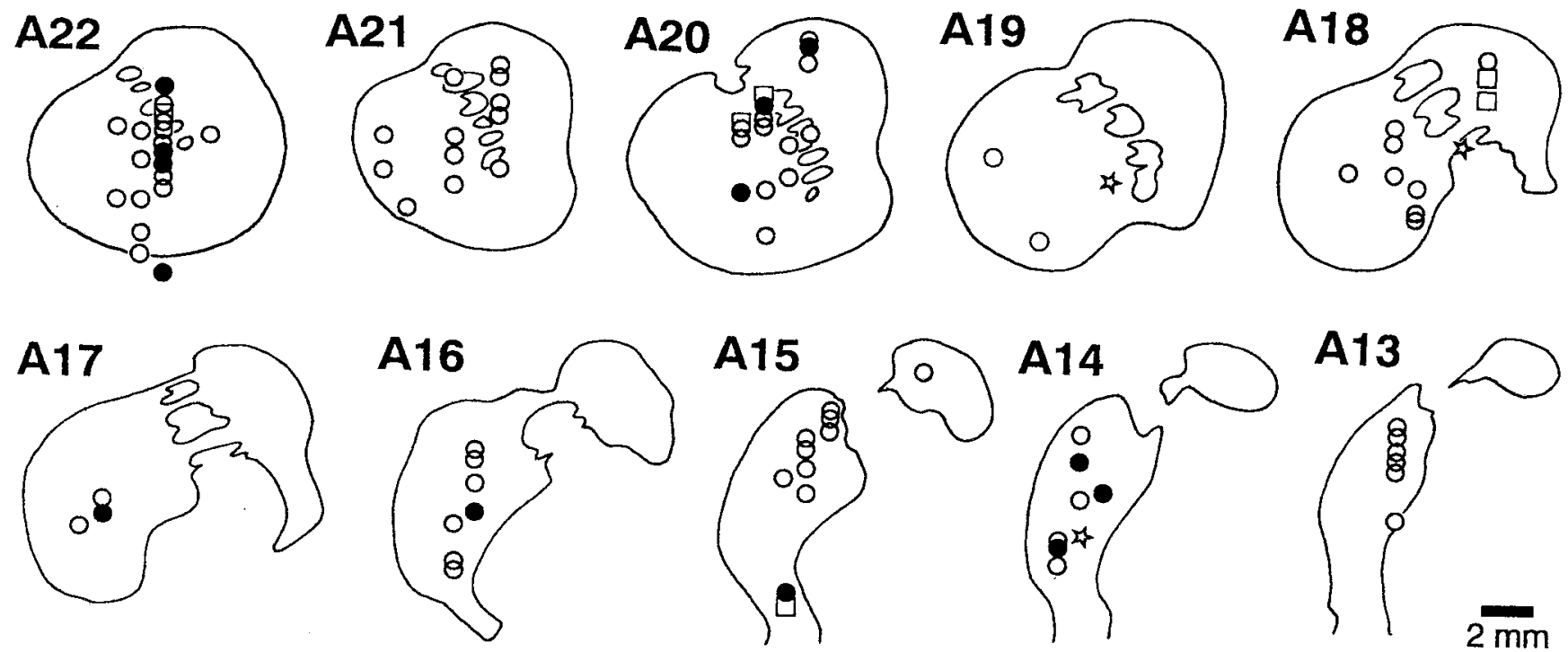
O loud sound and air puff + liquid reward
loud sound or air puff + liquid reward

\section{loud sound and/or air puff}

Figure 4. Positions of tonically active neurons with different response selectivities in the three monkeys. Recording sites of neurons tested with all three stimuli are plotted on standard coronal sections labeled in rostrocaudal stereotaxic planes. loud sound and airpuff + liquid reward, Responses to all three stimuli; loud sound or airpuff + liquid reward, neurons responding to the delivery of juice and to at least one of the aversive stimuli; loud sound and/or airpuff, selective responses to aversive stimuli; liquid reward, selective responses to the delivery of juice.

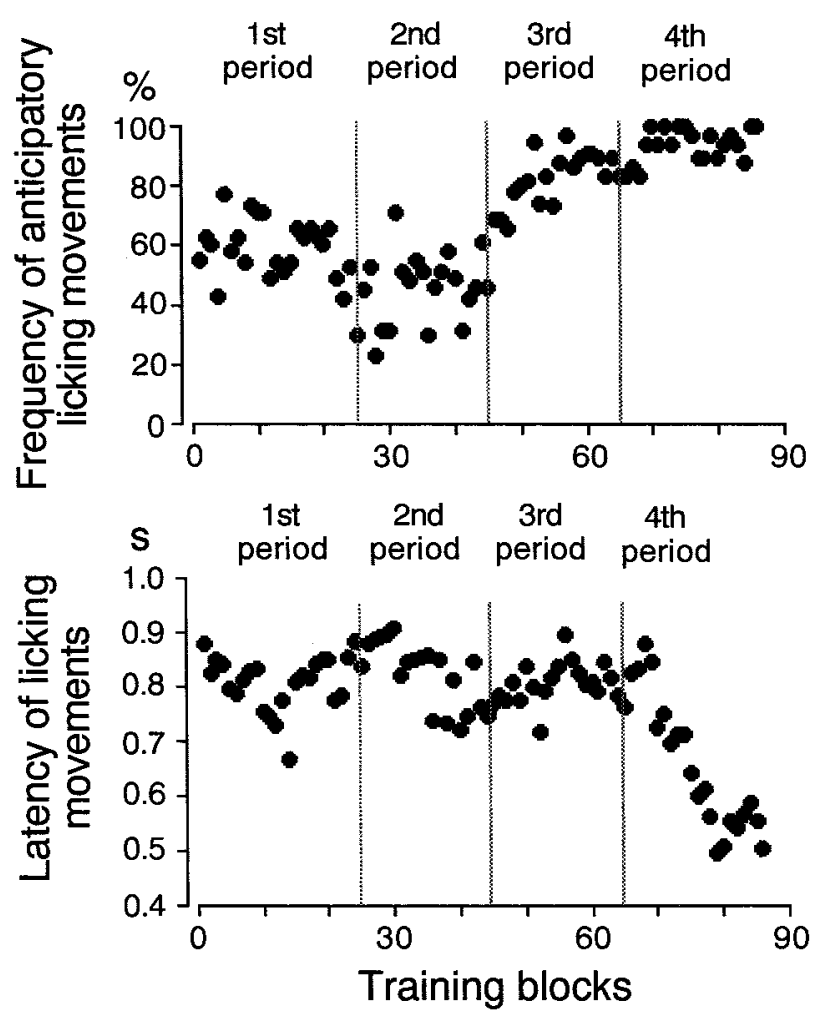

Figure 5. Learning curves for the stimulus-reward association on one monkey. Each point indicates the percentage of licks starting before reward delivery compared with the total number of trials by block (top) and the latency of licks (bottom) for each block.

sponse of TANs appears to depend on the novelty and intensity of the stimulus, suggesting that all stimuli that are effective in attracting attentional resources would elicit neuronal responses
(Aosaki et al. 1994b; Blazquez et al., 2002). However, one can assume that the neuronal modulation that occurs with different levels of attention to stimuli is typically a change in the magnitude of the neuronal response elicited by the stimulus rather than a change in the pattern of neuronal responding. Although the present evidence appears to show that the motivational value of stimuli is the relevant factor here, it is impossible to rule out the possibility that the TAN responses may be related to some behavioral reactions or attentional processes associated with stimulus detection. Additional exploration of these aspects involved in the sensitivity of TANs to environmental events is needed to provide a more complete explanation of the functional significance of the responses of these neurons.

TANs, learning, and motivation

It has been reported that the acquisition of TAN responses occurs when the motivational value of a stimulus was manipulated from neutral to appetitive (Aosaki et al., 1994b). Our findings go further to provide the first evidence for the capacity of these neurons to show response plasticity when the motivational value of sensory information undergoes changes into opposite directions during associative learning. Additionally, we observed that the change in the expression of TAN responses to the stimulus was paralleled by a lack of responsiveness to reward. We demonstrated previously that the response of TANs to an appetitive stimulus is reduced or suppressed when its timing can be predicted (Sardo et al., 2000; Ravel et al., 2001). Such an effect was confirmed here under a condition in which aversive stimuli gained appetitive qualities through repeated association with reward, thus adding a motivational integrative dimension to the involvement of TANs in predictive aspect of behavior.

Although recent neurophysiological experiments on monkeys have pointed out the importance of the reward context in the numerous forms of behavior-related activity in the striatum 


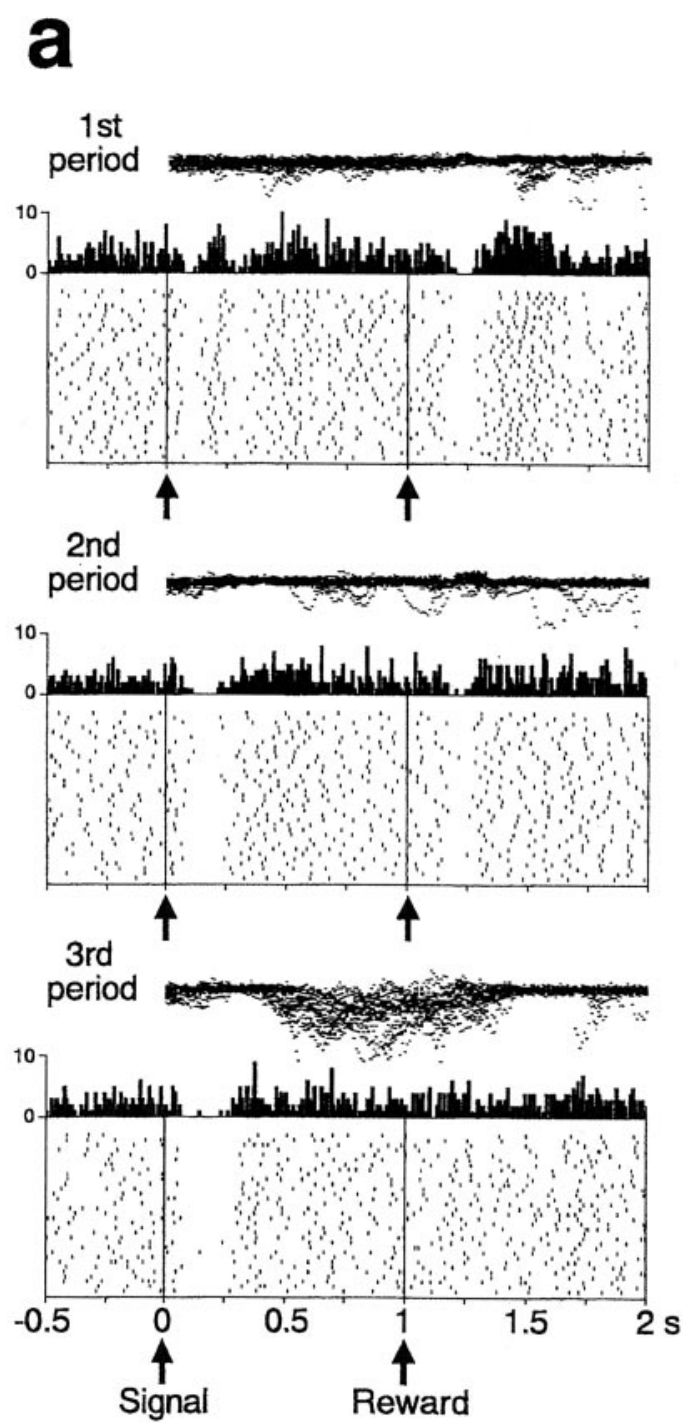

b

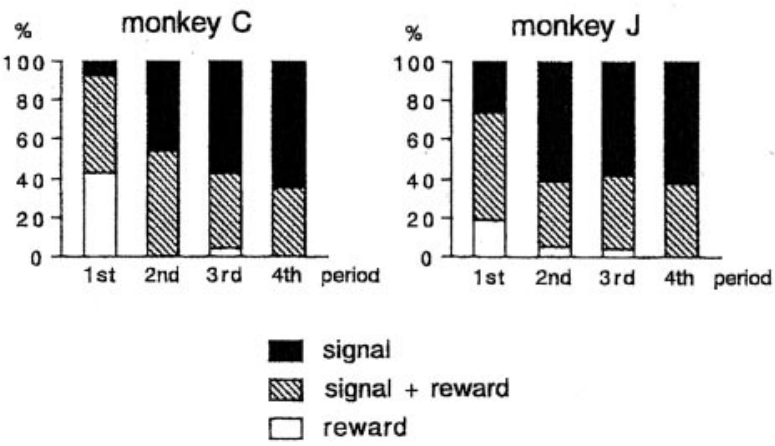

Figure 6. Neuronal correlates of learning of associations between aversive stimuli and reward. $a$, Changes of representative responses to the auditory stimulus and reward of three TANs recorded during successive training periods. Conventions are similar to those in Figure 1. Superimposed traces of licking movement records aligned on the onset of the auditory stimulus are shown at the top of each perievent histogram. $b$, Changes of the relative proportions of neurons responding to the auditory stimulus and/or reward for monkeys C and J.
(Hollerman et al., 1998; Kawagoe et al., 1998; Hassani et al., 2001; Takikawa et al., 2002), relatively few studies have investigated the participation of this structure in processes that involve aversive stimulus conditions (Rolls et al., 1983; Williams et al., 1993; White et al., 1994; Koyama et al., 2000). The results obtained here are consistent with data from previous studies showing the effectiveness of aversive events for modulating TANs (Ravel et al., 1999; Blazquez et al., 2002). Most of the neurons responding to aversive stimuli also responded to the liquid reward delivery, indicating that the striatum does not contain separate populations of TANs involved in the processing of information about pleasant and unpleasant stimuli. Thus, at the level of the TANs, both aspects of the information can be integrated, and the ability of these neurons to sample from a wide range of motivationally relevant stimuli may be crucial for their contributions to motor response selection functions. In contrast, the response of midbrain dopamine neurons to stimuli preferentially concerns appetitive rather than aversive motivational value, suggesting that these neurons mediate the rewarding effects of external stimuli that may contribute to mechanisms for reinforcement learning (Mirenowicz and Schultz, 1996; Schultz, 1998). Few TANs, however, were responding to only one type of stimulus, this selectivity being presumably based on the motivational value or the physical identity of the stimulus.

\section{Contrasting responses of TANs to motivationally opposing stimuli}

It has been considered that TANs correspond to cholinergic interneurons in the striatum (Kimura et al., 1990; Wilson et al., 1990; Aosaki et al., 1995). The observation that aversive stimuli did not elicit responses in the same manner as those elicited by appetitive stimuli might suggest that TANs deliver distinct signals to other types of striatal neurons, especially the GABAergic medium spiny projection neurons. These signals may correspond to phasic modulations of the general tone of cholinergic transmission in relation to the motivational content of stimuli. The discriminative responses of TANs may be ascribed to distinct inputs arising from afferent sources of the striatum. In particular, the phasic activations of dopamine neurons appear to be focused on appetitive stimulus properties (Mirenowicz and Schultz, 1996), and it has been proposed that the transient suppression in the tonic firing of TANs in response to reward-predicting stimuli is mediated by a dopaminergic mechanism (Bennett and Wilson, $1998,1999)$. Because it has been shown that the response of TANs to conditioned appetitive stimuli is dependent on the dopamine input to the striatum (Aosaki et al., 1994a; Raz et al., 1996; Watanabe and Kimura, 1998), an obvious possibility is that the reward value of stimuli could gain access to these neurons through ascending dopamine fibers. Other inputs from structures implicated in the evaluation of the motivational significance of stimuli, especially the orbitofrontal cortex and amygdala (Nishijo et al., 1988; Schoenbaum et al., 1998; Tremblay and Schultz, 1999), can also participate in shaping the contrasting responses of TANs for appetitive and aversive stimuli. However, it is notable that the presently observed neuronal responses were not located in the ventral part of the striatum, which is relatively specialized for processing motivational information arising from limbic structures (Russchen et al., 1985; Haber et al., 1995; Fudge et al., 2002). Another possible source of the responses of TANs is the thalamus because anatomical studies have shown that striatal cholinergic interneurons are contacted preferentially by fibers from the intralaminar thalamic nuclei (Lapper and Bolam, 1992; Sidibé and Smith, 1999). It has been reported that inputs from these tha- 

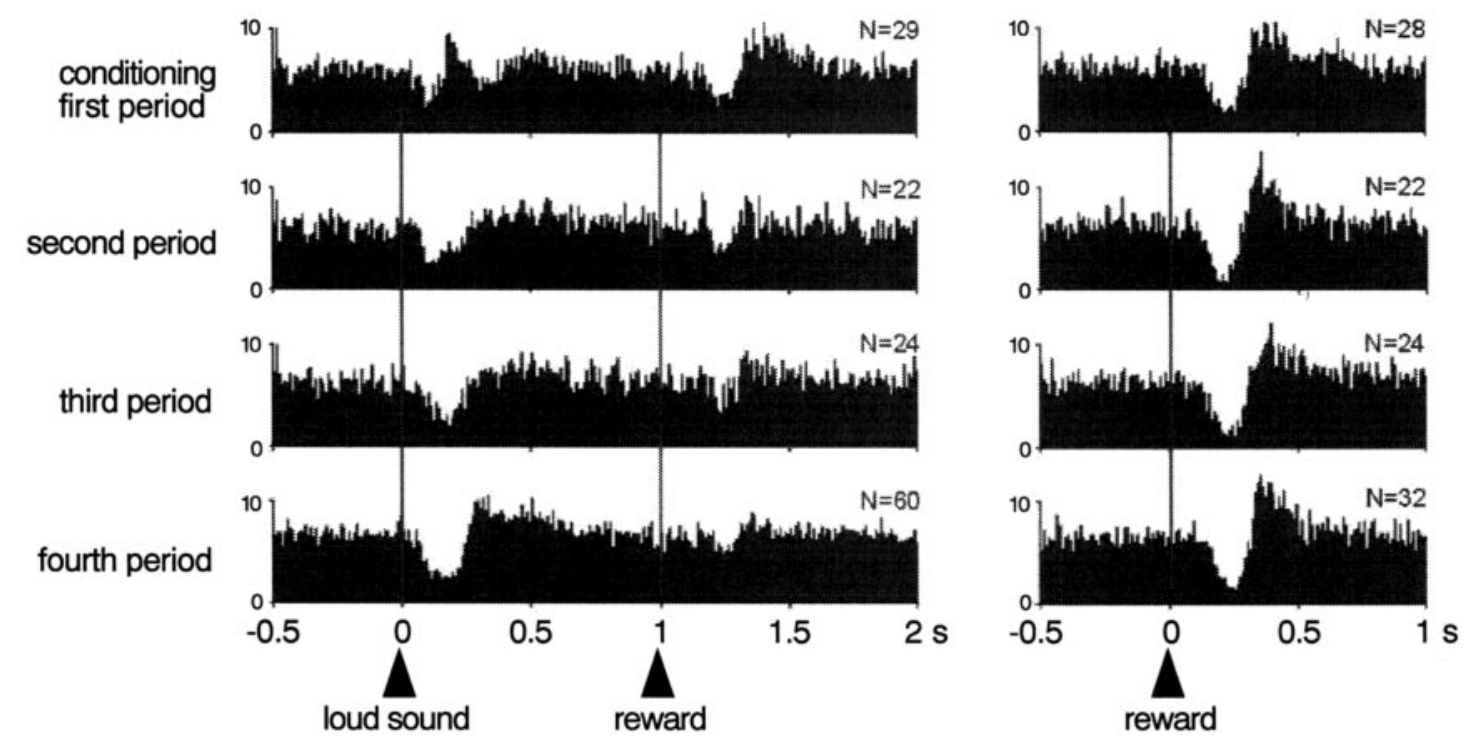

Figure 7. Changes of population responses of TANs during the learning of the stimulus-reward association. Histograms show, for one monkey, neuronal data from the four phases of training (left) and from separate blocks of trials in which the liquid reward was delivered alone (right). Data were taken from the same neurons tested in both the conditioning procedure and outside of the task, with the exception of the first and fourth periods. Vertical scale indicates mean impulse rate. N, Number of neurons included for each population histogram.

lamic areas can modulate the responsiveness of TANs to conditioned appetitive stimuli (Matsumoto et al., 2001), but it is not known whether TAN responses to aversive stimuli are also dependent on these inputs. Although the basis of differential TAN responses has yet to be identified in detail, it is not clear how changes in the pattern of TAN responding could provide a signal required to adjust the subsequent action selection. One could hypothesize that a motivation-dependent change of cholinergic tone, mediated by the precise profile of change in the firing of TANs, could selectively influence the striatal output circuits involved in generating behavioral reactions to motivationally opposing stimuli. A major emphasis of work on the striatum and ascending midbrain dopamine projections has been on the processing of stimuli that are primarily rewarding or by signals that predict their occurrence. The present study suggests that the striatum is also a component of the neural circuitry mediating behavioral responses elicited by aversive events. By virtue of their sensitivity to motivationally opposing stimuli, TANs are in a position to process the information necessary to select actions in accordance with the animal's motivational state. Their discriminative responses may play a role in relaying information about the specific motivational properties of sensory events to output striatal systems. Such responses would allow TANs to participate in the mechanisms subserving the ability to adjust the behavior to primary motivating events and associations formed during learning.

\section{References}

Aosaki T, Graybiel AM, Kimura M (1994a) Effect of the nigrostriatal dopamine system on acquired neural responses in the striatum of behaving monkeys. Science 265:412-415.

Aosaki T, Tsubokawa H, Ishida A, Watanabe K, Graybiel AM, Kimura M (1994b) Responses of tonically active neurons in the primate's striatum undergo systematic changes during behavioral sensorimotor conditioning. J Neurosci 14:3969-3984.

Aosaki T, Kimura M, Graybiel AM (1995) Temporal and spatial characteristics of tonically active neurons of the primate's striatum. J Neurophysiol 73:1234-1252.

Apicella P, Ljungberg T, Scarnati E, Schultz W (1991) Responses to reward in monkey dorsal and ventral striatum. Exp Brain Res 85:491-500.

Apicella P, Scarnati E, Ljungberg T, Schultz W (1992) Neuronal activity in monkey striatum related to the expectation of predictable environmental events. J Neurophysiol 68:945-960.

Apicella P, Legallet E, Trouche E (1997) Responses of tonically discharging neurons in the monkey striatum to primary rewards delivered during different behavioral states. Exp Brain Res 116:456-466.

Bennett BD, Wilson CJ (1998) Synaptic regulation of action potential timing in neostriatal cholinergic interneurons. J Neurosci 18:8539-8549.

Bennett BD, Wilson CJ (1999) Spontaneous activity of neostriatal cholinergic interneurons in vitro. J Neurosci 19:5586-5596.

Blazquez PM, Fujii N, Kojima J, Graybiel AM (2002) A network representation of response probability in the striatum. Neuron 33:973-982.

Bowman EM, Aigner TG, Richmond BJ (1996) Neural signals in the monkey ventral striatum related to motivation for juice and cocaine rewards. J Neurophysiol 75:1061-1073.

Fudge JL, Kunishio K, Walsh P, Richard C, Haber SN (2002) Amygdaloid projections to ventromedial striatal subterritories in the primate. Neuroscience 110:257-275.

Haber SN, Kunishio K, Mizobuchi M, Lynd-Balta E (1995) The orbital and medial prefrontal circuit through the primate basal ganglia. J Neurosci 15:4851-4867.

Hassani OK, Cromwell HC, Schultz W (2001) Influence of expectation of different rewards on behavior-related neuronal activity in the striatum. J Neurophysiol 85:2477-2489.

Hikosaka O, Sakamoto M, Usui S (1989) Functional properties of monkey caudate neurons. III. Activities related to expectation of target and reward. J Neurophysiol 61:814-832.

Hikosaka O, Rand MK, Miyachi S, Miyashita K (1995) Learning of sequential movements in the monkey: process of learning and retention in memory. J Neurophysiol 74:1652-1661.

Hollerman JR, Tremblay L, Schultz W (1998) Influence of reward expectation on behavior-related neuronal activity in primate striatum. J Neurophysiol 80:947-963.

Kawagoe R, Takikawa Y, Hikosaka O (1998) Expectation of reward modulates cognitive signals in the basal ganglia. Nat Neurosci 1:411-416.

Kimura M, Kato M, Shimazaki H (1990) Physiological properties of projection neurons in the monkey striatum to the globus pallidus. Exp Brain Res 82:672-676.

Koyama T, Kato K, Mikami A (2000) During pain-avoidance neurons activated in the macaque anterior cingulate and caudate. Neurosci Lett 283:17-20

Lapper SR, Bolam JP (1992) Input from the frontal cortex and the parafascicular nucleus to cholinergic interneurons in the dorsal striatum of the rat. Neuroscience 51:533-545.

Matsumoto N, Minamimoto T, Graybiel AM, Kimura M (2001) Neurons in the thalamic CM-Pf complex supply striatal neurons with information 
about behaviorally significant sensory events. J Neurophysiol 85:960-976.

Mirenowicz J, Schultz W (1996) Preferential activation of midbrain dopamine neurons by appetitive rather aversive stimuli. Nature 379:449-451.

Nishijo H, Ono T, Nishino H (1988) Single neuron responses in amygdala of alert monkey during complex sensory stimulation with affective significance. J Neurosci 8:3570-3583.

Parent A (1990) Extrinsic connections of the basal ganglia. Trends Neurosci 13:254-258.

Ravel S, Legallet E, Apicella P (1999) Tonically active neurons in the monkey striatum do not preferentially respond to appetitive stimuli. Exp Brain Res 128:531-534.

Ravel S, Sardo P, Legallet E, Apicella P (2001) Reward unpredictability inside and outside of a task context as a determinant of the responses of tonically active neurons in the monkey striatum. J Neurosci 21:5730-5739.

Raz A, Feingold A, Zelanskaya V, Vaadia E, Bergman H (1996) Neuronal synchronization of tonically active neurons in the striatum of normal and parkinsonian primates. J Neurophysiol 76:2083-2088.

Rolls ET, Thorpe SJ, Maddison SP (1983) Responses of striatal neurons in the behaving monkey. I. Head of the caudate nucleus. Behav Brain Res 7:179-210.

Russchen FT, Bakst I, Amaral DG, Price JL (1985) The amygdalostriatal projections in the monkey. An anterograde tracing study. Brain Res 329:241-257.

Sardo P, Ravel S, Legallet E, Apicella P (2000) Influence of the predicted time of stimuli eliciting movements on responses of tonically active neurons in the monkey striatum. Eur J Neurosci 12:1801-1816.

Schoenbaum G, Chiba AA, Gallagher M (1998) Orbitofrontal cortex and basolateral amygdala encode expected outcome during learning. Nat Neurosci 1:155-159.

Schultz W (1998) Predictive reward signal of dopamine neurons. J Neurophysiol 80:1-27.

Schultz W, Apicella P, Scarnati E, Ljungberg T (1992) Neuronal activity in monkey ventral striatum related to the expectation of reward. J Neurosci 12:4595-4610.

Shidara M, Aigner T, Richmond BJ (1998) Neuronal signals in the monkey ventral striatum related to progress through a predictable series of trials. J Neurosci 18:2613-2625.

Sidibé M, Smith Y (1999) Thalamic inputs to striatal interneurons in monkeys: synaptic organization and co-localization of calcium binding proteins. Neuroscience 89:1189-1208.

Takikawa Y, Kawagoe R, Hikosaka O (2002) Reward-dependent spatial selectivity of anticipatory activity in monkey caudate nucleus. J Neurophysiol 87:508-515.

Tremblay L, Schultz W (1999) Relative reward preference in primate orbitofrontal cortex. Nature 398:704-708.

Watanabe K, Kimura M (1998) Dopamine receptor-mediated mechanisms involved in the expression of learned activity of primate striatal neurons. J Neurophysiol 79:2568-2580.

White IM, Miller DP, White W, Dike GL, Rebec GV, Steinmetz JE (1994) Neuronal activity in rabbit neostriatum during classical eyelid conditioning. Exp Brain Res 99:179-190.

Williams GV, Rolls ET, Leonard CM, Stern C (1993) Neuronal responses in the ventral striatum of the behaving macaque. Behav Brain Res 55:243-252.

Wilson CJ, Chang HT, Kitai ST (1990) Firing patterns and synaptic potentials of identified giant aspiny interneurons in the rat neostriatum. J Neurosci 10:508-519. 